\title{
Implementación y análisis de un patio científico en la etapa de Educación Infantil
}

Recepción: 30/11/2020 | Revisión: 08/02/2021 | Aceptación: 09/08/2021 | Preprint: 01/10/2021 | Publicación: 01/03/2022

Raul PEINADO ALAMILLO

Universitat de Lleida

raulpeinadoalamillo@gmail.com

https://orcid.org/ oooo-0001-9266-4821

Anna SOLÉ LLUSSÀ

Universitat de Lleida

anna.sole@udl.cat

http://orcid.org/oooo-00o3-2921-5956

\author{
iD David AGUILAR CAMAÑO \\ Universitat de Lleida \\ david.aguilar@udl.cat \\ http://orcid.org/oooo-0oo1-5940-3339
}

\begin{abstract}
Resumen: La realidad actual en la etapa de educación infantil pone de manifiesto dos aspectos que han motivado la elaboración del presente estudio. En primer lugar, los patios escolares no cuentan con recursos que promuevan el aprendizaje, y, en segundo lugar, la ciencia se contempla como algo inabarcable y lejano en estas edades. Por estos motivos, el presente trabajo propone diseñar diferentes propuestas de carácter científico para implementarlas en el patio escolar de una escuela catalana y analizar la interacción de 70 escolares con edades comprendidas entre 3 y 5 años con este tipo de recursos. A través de un estudio cualitativo basado en observaciones no participantes del contexto en el que se ha intervenido, se ha evidenciado cómo se puede impulsar este espacio escolar, abasteciéndolo con propuestas de libre funcionamiento que contribuyen a la inclusión, a la generación de experiencias gratificantes y al desarrollo de las habilidades científicas entre alumnos de educación infantil.
\end{abstract}

Palabras clave: ciencia; patio científico; habilidades científicas; experimentación; educación infantil.

\section{IMPLEMENTATION AND ANALYSIS OF A SCIENTIFIC SCHOOLYARD IN EARLY CHILDHOOD EDUCATION}

\begin{abstract}
The current state of early childhood education reveals two aspects which have motivated this study. Firstly, schoolyards do not usually have resources which promote learning, and, secondly, science is seen as something immeasurable and distant at this education stage. For these reasons, the present study aims to design different scientific proposals to be implemented in the schoolyard of a Catalan school and to analyze how children between 3 and 5 years of age interact and learn with them. Through this qualitative study, based on non-participant observations of the context, it is shown how this school space can be stimulated by supplying proposals which students can freely explore. These proposals contribute to inclusion, to the generation of positive experiences and to the development of scientific skills among early childhood education students.
\end{abstract}

Keywords: science, scientific schoolyard; scientific skills; experimentation; early childhood education. 


\section{IMPLEMENTACIÓ I ANÀLISI D'UN PATI CIENTÍFIC EN L'ETAPA D'EDUCACIÓ INFANTIL}

Resum: La realitat actual en l'etapa d'educació infantil posa de manifest dos aspectes que han motivat l'elaboració d'aquest estudi. En primer lloc, els patis escolars no tenen recursos que promoguin l'aprenentatge $i$, en segon lloc, la ciència es contempla com quelcom inabastable i llunyà en aquestes edats. Per aquestes raons, presentem diferents propostes de caràcter científic per implementar-les al pati escolar d'una escola i analitzar la interacció de 70 escolars d'entre 3 i 5 anys amb aquest tipus de recursos. A través d'un estudi qualitatiu basat en observacions no participants del context en el que s'hi ha intervingut, s'ha evidenciat com es pot impulsar aquest espai escolar, abastint-lo amb propostes de funcionament lliure que contribueixen a la inclusió, la generació d'experiències gratificants $i$ al desenvolupament de les habilitats científiques en alumnes d'educació infantil.

Paraules clau: ciència; pati científic; habilitats científiques; experimentació; educació infantil.

\section{Introducción}

Los profesionales del ámbito educativo reconocen a los niños y niñas como seres inteligentes, capaces, competentes, constructores de conocimiento, identidad y cultura que desarrollan ideas y teorías que examinan y reelaboran a partir de sus experiencias y de la interacción con los demás (Hoyuelos, 2004; Malaguzzi, 2005; Pedreira y Márquez, 2017a). Los niños y niñas, prácticamente desde su nacimiento, tienen una manera única de explorar y conocer el mundo que les rodea. Esta interacción con el entorno es un aspecto fundamental de su aprendizaje ya que les permite adquirir ideas e imaginar modelos que les puedan ser útiles para interpretar y comprender los fenómenos y procesos que tienen lugar en el mundo (Eshach y Fried, 2005; Fleer y Hardy, 2001; Rinaldi, 2006). De hecho, se entiende la ciencia como una actividad humana presente en la cotidianidad y que es clave para conocer, comprender y apreciar el mundo donde vivimos (Pujol, 2003; Quintanilla et al., 2011). Por tanto, parece importante aportar ayudas y soportes al infante para que pueda estructurar e impulsar este tipo de acciones exploratorias y, así, fomentar el desarrollo de un conocimiento científico útil que les permita entender mejor su realidad cercana (Osborne, 2014). De esta manera, y según destacan informes de expertos españoles relacionados con la enseñanza de las ciencias como el informe Enciende (COSCE, 2011), el aprendizaje científico es necesario impulsarlo desde el inicio de la vida escolar de los niños y niñas, ofreciendo experiencias positivas y de calidad que favorezcan: i) el aprendizaje sobre el mundo; ii) la reflexión crítica; iii) la habilidad de investigar; y iv) la curiosidad y el desarrollo de una actitud favorable hacia temas científicos (Eshach y Fried, 2005; Gopnik y Wellman, 2012; Kloos et al., 2018; Liguori y Noste, 2007).

Diferentes autores afirman que el desarrollo del conocimiento científico en educación infantil implica comenzar a sistematizar y a estructurar las acciones exploratorias para descubrir el funcionamiento de objetos y fenómenos que rodean al estudiante. Esto conlleva ayudar al alumnado a que pueda explicar y argumentar las evidencias que recoge de su entorno, utilizar instrumentos cuando sea necesario, empezar a poner nombres, ordenar, clasificar, comparar, dialogar, etc. Es decir, todo un conjunto de habilidades que implican una manera de pensar muy concreta y asociada al pensamiento científico y que ayudarán al estudiante de infantil a cuestionarse sobre su entorno y a construir nuevas ideas al respecto (Harlen, 2010; Osborne, 2014; Saçkes, 2014). Este conjunto de habilidades se desarrolla de manera interrelacionada durante el proceso de aprendizaje científico y son enfocadas y organizadas de 
manera ligeramente diferente según la tradición cultural e investigadora, pero manteniendo grandes coincidencias. Pedreira y Márquez (2016, 2017a, 2017b) realizan una adaptación, dirigida a las primeras edades, de este conjunto de habilidades que forman parte del aprendizaje científico y las organiza según el objetivo de las mismas en las siguientes tres fases:

i. Experiencia: relacionada con las acciones y habilidades que hacen referencia al contacto directo con el entorno. Es decir, es el momento en el que el estudiante explora y experimenta con la realidad: observa, manipula, usa instrumentos (balanzas, lupas), etc.

ii. Explicitación: hace referencia a la comunicación verbal o no verbal de las ideas que tienen los alumnos, empleando habilidades cognitivas (clasificar, ordenar, comparar, aparejar, etc.) o cognitivo-lingüísticas (describir, explicar, nombrar, etc.).

iii. Evolución: incluye las habilidades que demuestran que el estudiante ha introducido cambios en su manera de pensar, mostrando una evolución de sus ideas y una tendencia hacia la modelización, es decir, hacia el inicio de la integración de teorías e ideas procedentes de la ciencia. Este cambio puede evidenciarse, por ejemplo, mediante la formulación de nuevas preguntas, estableciendo relaciones con ideas anteriores, etc. También es fundamental destacar la importancia de la interacción entre iguales o con adultos, hecho que permite aportar nuevas perspectivas y contribuir a dicha evolución de las ideas.

Asimismo, diversos estudios indican que el aprendizaje científico en esta etapa educativa también ha de ir acompañado de experiencias que impulsen el entusiasmo, el interés y la motivación por los fenómenos que forman parte de nuestro entorno (Hein, 2009). Es importante que las escuelas traten de promover la curiosidad del alumno por el mundo que les rodea, el placer y la emoción por el aprendizaje científico (Harlen, 2010).

Pero este conjunto de elementos, identificados como necesarios en el aprendizaje de las ciencias, no suelen ser habituales en las aulas de educación infantil. El alumnado de dicha etapa educativa no acostumbra a estar familiarizados con las ciencias puesto que éstas no reciben tanta atención como otros ámbitos de conocimiento tales como la lectura o las matemáticas (Gómez-Motilla y Ruiz-Gallardo, 2016; Mantzicopoulos et al., 2008). Además, se sigue observando cómo el aprendizaje de las ciencias se desarrolla a través de fichas o de libros de texto, hecho que no ofrece ningún tipo de experiencia directa con la realidad, no promueve el pensamiento divergente de los infantes y no da valor a las ideas de los mismos (Pedreira y Márquez, 2016).

Sin embargo, la introducción del aprendizaje por ambientes o espacios ha proporcionado un impulso a las ciencias en educación infantil. Un espacio de aprendizaje se entiende como un entorno dinámico en el que se generan intencionalmente un conjunto de actividades y acciones que permiten conseguir un objetivo de aprendizaje determinado y el desarrollo competencial en una o varias áreas de conocimiento (Castro-Pérez y Morales-Ramírez, 2015; Epstein, 2007; Sevilla, 2010). Este tipo de espacios sitúan al estudiante como protagonista de su proceso de aprendizaje y son generadores de situaciones que invitan al infante a imaginar, explorar, diseñar y transformar su entorno (Ferreira et al., 2018; National Learning Infraestructure Initiative, 2004; Riera et al., 2014). Concretando en los espacios de aprendizaje científicos para 
educación infantil, Pedreira y Márquez (2016, 2017a) indican que estos entornos han de ser de libre elección y han de fomentar la experimentación directa con materiales y fenómenos, impulsando el interés del estudiante por las ciencias. Los espacios científicos han de ofrecer recursos y propuestas que favorezcan procesos de descubrimiento e investigación relacionados con algún ámbito de las ciencias y que promuevan el desarrollo de habilidades científicas. Estas propuestas han de presentar una intencionalidad clara y definida de aprendizaje, pero a la vez han de ser abiertas para permitir que tengan lugar acciones no previstas. Hasta el momento, se han publicado pocas investigaciones que analicen sistemáticamente los procesos de aprendizaje que emergen en espacios científicos orientados a educación infantil. Estos estudios indican que los espacios de ciencias: i) son capaces de ofrecer experiencias gratificantes y de bienestar al alumnado: ii) favorecen el desarrollo de diferentes habilidades científicas, principalmente las relacionadas con la experiencia directa con el entorno, la explicitación de ideas y la generación de preguntas (Pedreira y Márquez, 2017a, 2017b).

A lo largo de los últimos años, se ha observado un incremento en el interés de este tipo de espacios de ciencia en educación infantil. Se observa cómo se han realizado organizaciones espacio-temporales en escuelas de manera que, en momentos puntuales y a través de sesiones programadas, los infantes dejan su aula habitual y acceden a este tipo de espacios. A veces, el alumnado también acude a museos o centros de educación no formales que ofrecen este tipo de ambientes de aprendizaje (Lemkow et al., 2016; Pedreira y Márquez, 2017b). En vista de los beneficios que proporcionan los espacios de ciencia, parece interesante buscar nuevas estrategias para impulsar el uso dichos espacios de aprendizaje en la escuela y, en consecuencia, potenciar el aprendizaje científico en educación infantil. Encontrar nuevos escenarios escolares que faciliten un acceso más continuado a espacios de dicha tipología, yendo más allá de un conjunto de sesiones programadas durante el horario lectivo, podría ser una posibilidad.

Por ejemplo, el patio escolar es un entorno al que los estudiantes acceden diariamente y cuyo potencial educativo en el ámbito de las ciencias no siempre se explota (Ayotte-Beaudet et al., 2017; Christidou et al., 2013). Muchos patios escolares presentan diseños fijos que presentan usos muy limitados para, así, mantener el control de las actividades que realiza el alumnado (Gallardo y Turkienicz, 2018). Se suelen observar patios limpios y ordenados en los que se tiende a reproducir roles, relaciones de poder y desigualdad sexual ya que ni los recursos ni el espacio se distribuyen equitativamente entre niños y niñas y también porque los juegos que desarrollan unos y otros suelen diferenciarse por género (Saldaña, 2018; Subirats y Tomé, 2010). Hasta el momento, la mayoría de los trabajos que han estudiado los beneficios del patio escolar en cuanto al aprendizaje de las ciencias se han centrado en el uso de dicho escenario como recurso o espacio donde desarrollar actividades científicas puntuales principalmente en educación primaria y secundaria (por ejemplo, Atmodiwirjo, 2013; Ayotte-Beaudet et al., 2017; Ting y Siew, 2014). Sin embargo, existe un vacío en la literatura de trabajos focalizados en educación infantil y, especialmente, enfocados en el rediseño de entornos escolares tradicionales como el patio para potenciar el aprendizaje científico. El hecho de incluir elementos pedagógicos propios de los espacios o ambientes de ciencias en el patio escolar podría ayudar a diversificar el tipo de actividades que se desarrollan en él a la vez que aportar nuevas posibilidades de aprendizaje para el alumnado de educación infantil. De esta manera, el estudiante entraría en contacto de manera más regular con diferentes materiales y propuestas de carácter científico con las que interactuaría de manera libre y autónoma durante su tiempo de recreo, impulsándose un modelo de aprendizaje similar al que se encuentra en los espacios de ciencias de libre elección. 
En el presente trabajo, se diseñarán e instalarán recursos y propuestas de carácter científico de manera permanente en el patio de una escuela de educación (estudiantes de 3 a 5 años). Se trata de un estudio preliminar en el que se analizarán las experiencias y procesos de aprendizaje científicos promovidos por la instalación de dicho material en el patio. Por un lado, este artículo aportará nuevos datos que complementarán a la escasez de investigaciones sobre el tipo de aprendizaje que emerge en espacios de ciencias de libre elección dedicados a educación infantil. Y, por otro lado, también puede aportar nuevas ayudas pedagógicas para impulsar el aprendizaje científico en entornos no formales como el patio escolar.

De esta manera, los objetivos del presente trabajo son los siguientes:

\begin{abstract}
A. Analizar el tipo de actividades y comportamiento que el alumnado de educación infantil presenta en el patio escolar antes y después de instalar los recursos y propuestas científicas. Se atenderá a posibles diferencias en función de la edad y del género.
\end{abstract}

B. Analizar el desarrollo de experiencias de aprendizaje positivas y el desarrollo de habilidades científicas en el alumnado de educación infantil que participa en las propuestas científicas.

\title{
1. Metodología \\ 1.1 Diseño de investigación
}

El artículo presente se trata de un estudio de caso con carácter exploratorio en el que se aplica un método cualitativo para abordar los objetivos propuestos. Los datos cualitativos se obtienen a partir de observaciones de la práctica de las estudiantes realizadas en el propio contexto natural, el patio escolar (Bisquerra, 2004; Rennie y Johnston, 2004).

\subsection{Participantes}

Los sujetos han sido 70 escolares (33 niñas y 37 niños) de educación infantil con edades comprendidas entre los 3 y los 5 años. Los estudiantes pertenecen a una escuela pública urbana de Cataluña (este de España). Dichos participantes proceden de familias con un estatus socio-económico medio y siguen el currículum establecido por el Departamento de Educación (Gobierno de Cataluña) para educación infantil. Los estudiantes participantes no disponen de experiencia previa en espacios de aprendizaje científicos de libre elección.

\subsection{Diseño del patio escolar}

La Figura 1 muestra un plano del patio escolar en el que se ha intervenido. Éste presenta una amplia zona central destinada principalmente al desarrollo de juegos deportivos, un área recreativa (con mesas y juegos tradicionales pintados en el suelo), un arenero y una zona con vegetación a su alrededor. Además, los estudiantes disponen de material que pueden utilizar libremente como, por ejemplo, balones y bicicletas. 


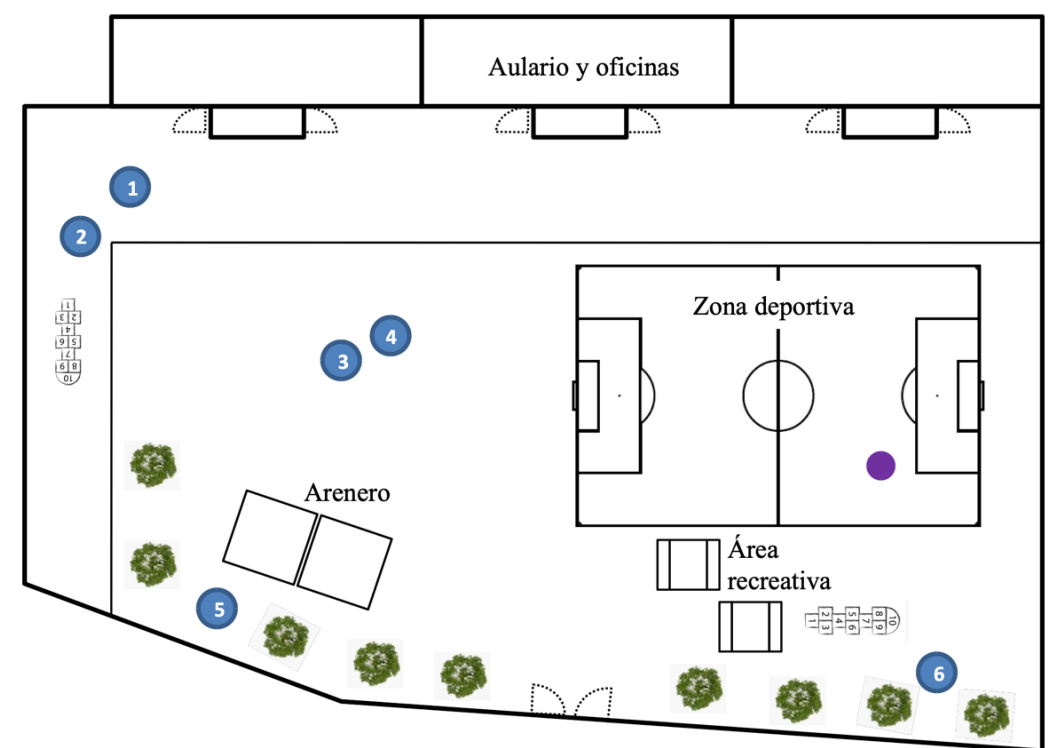

Figura 1. Plano del patio escolar analizado.

Los puntos 1 - 6 de la Figura 1 muestran la ubicación de las diferentes nuevas instalaciones realizadas en el patio escolar. Estas instalaciones presentan recursos y propuestas científicas con una tipología similar a la que se encuentran en los espacios de aprendizaje de ciencias de libre elección dirigidos a estudiantes de educación infantil. El diseño y elaboración de dichas propuestas fue realizado por dos investigadores del ámbito de la didáctica de las ciencias experimentales y por tres docentes del centro escolar, siguiendo los ejemplos descritos por Lemkow et al. (2016), Pedreira (2013) y Pedreira y Márquez $(2016,2017$ a) para preparar espacios de ciencia de libre elección infantiles. La Tabla 1 resume los objetivos y características de las seis propuestas instaladas.

\begin{tabular}{|l|l|l|}
\hline \multicolumn{2}{|c|}{ Descripción de las propuestas } \\
\hline $\begin{array}{l}\text { Propuesta 1: } \\
\text { Sonido }\end{array}$ & $\begin{array}{l}\text { Descripción: Se presentan doce botellas de } \\
\text { plástico iguales que contienen diferentes tipos } \\
\text { de semillas y frutos en su interior. Once de las } \\
\text { botellas son transparentes y es posible vislum- } \\
\text { brar su contendido. En cambio, una de las bo- } \\
\text { tellas es opaca. El alumnado puede interactuar } \\
\text { con las botellas, descubriendo y comparando el } \\
\text { sonido que se produce cuando éstas se agitan. } \\
\text { Objetivo científico: Comprobar la relación existente } \\
\text { entre el material con el que se rellena cada botella, } \\
\text { el tono y el volumen del sonido producido al agitar. }\end{array}$ \\
& \\
&
\end{tabular}




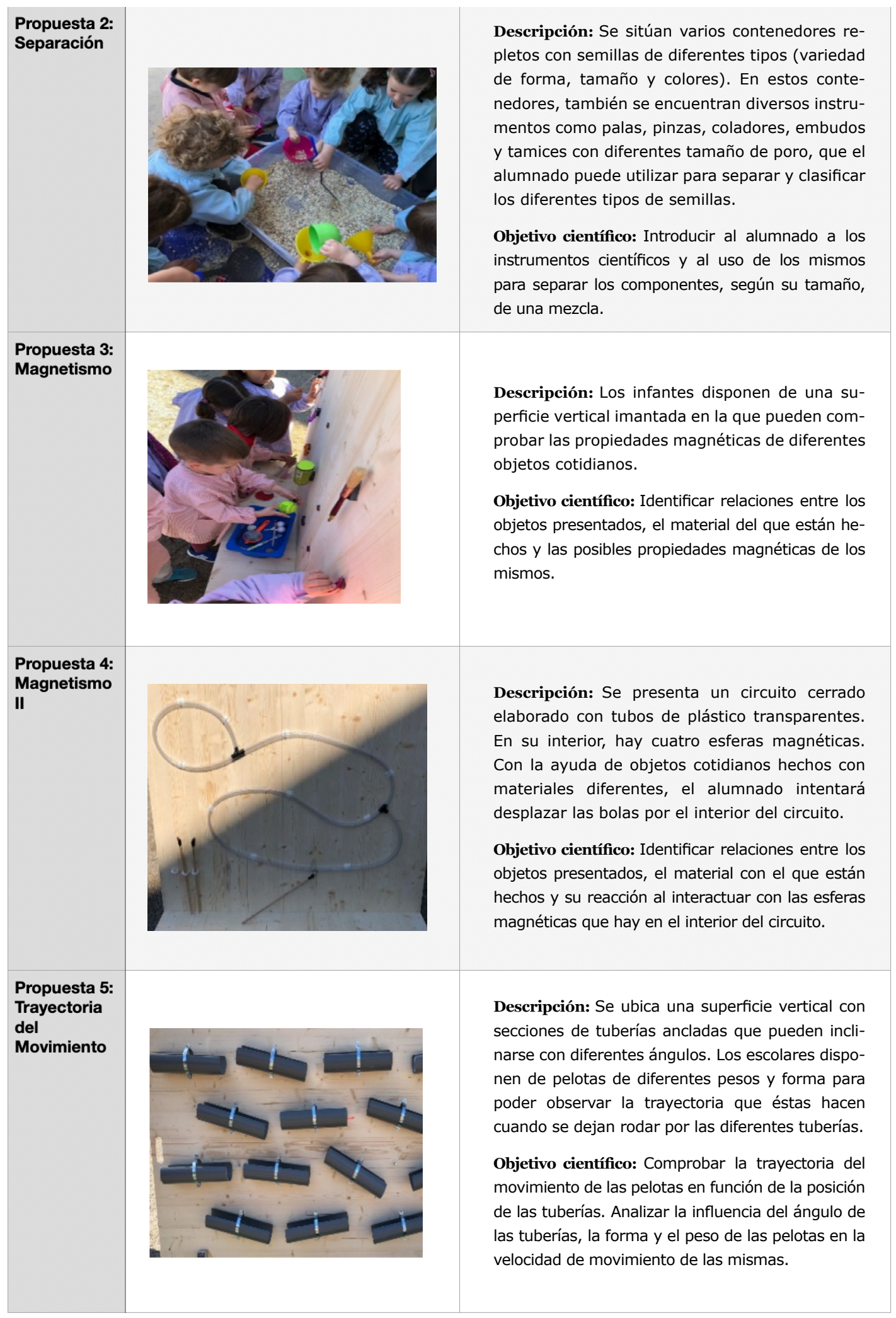




$\begin{aligned} & \text { Propuesta 6: } \\ & \text { Circuito de } \\ & \text { agua }\end{aligned}$
$\begin{aligned} & \text { Descripción: En una superficie vertical, se presenta un } \\ & \text { circuito elaborado con tubos de plástico que presenta } \\ & \text { diferentes llaves de paso. Los escolares pueden hacer } \\ & \text { circular el agua que se encuentra en un depósito co- } \\ & \text { nectado al circuito así como abrir o cerrar las llaves de } \\ & \text { paso para modificar la dirección del flujo de agua. Con } \\ & \text { la ayuda de una bomba hidráulica, los escolares pue- } \\ & \text { den retornar el agua del circuito al depósito. } \\ & \text { Objetivo científico: Comprobar cómo el agua tiende a } \\ & \text { fluir hacia abajo a través de un canal abierto. Probar el } \\ & \text { mecanismo de las llaves de paso y cómo éstas permiten } \\ & \text { bloquear el flujo de agua o cambiar su dirección }\end{aligned}$

Tabla 1. Características y objetivos de aprendizaje científico de las diferentes propuestas.

\subsection{Instrumentos de recogida de datos}

Para responder a los objetivos propuestos, se han realizado observaciones no participantes en el patio escolar. Estas observaciones tienen lugar durante el tiempo de recreo que los estudiantes tienen durante la mañana (40 minutos). En concreto, el estudio tiene lugar durante tres días consecutivos previos a la intervención en el patio escolar, para identificar las actividades y comportamientos habituales de los estudiantes en el patio y, a continuación, durante los siguientes doce días tras la instalación de las propuestas científicas. Durante dichos días, se hacen observaciones el primero, tercero, quinto i doceavo día.

La correspondiente recogida de datos se realiza a partir de notas de campo tomadas por un investigador y una investigadora y de registros videográficos realizados en el patio escolar.

Con respecto al objetivo $A$, se identificará el tipo de acciones y actividades que tienen los estudiantes previamente a la instalación de las propuestas científicas en el patio escolar. Para llevar a cabo esta parte del estudio, se adoptará una visión macroscópica, centrándose en "qué acción y dónde sucede" y no realizando un seguimiento concreto de estudiantes en particular (Christidou et al., 2013). Las actividades o comportamientos observados se codifican siguiendo las siguientes categorías: actividad física (jugar libremente con equipamiento -por ejemplo, utilizar las bicicletas disponibles, balones-, jugar en las estructuras fijas disponibles en el área recreativa), social (dialogar entre compañeros, observar la acción de otros compañeros, juegos de rol, creativos, imaginativos) o cognitivos (exploración del entorno, realizar construcciones en la zona de arena) (Christidou et al., 2013; Dyment y O'Connell, 2013; Tranter y Malone, 2014). A continuación, y a partir de la instalación de las propuestas científicas, se estudiarán posibles cambios en los comportamientos y actividades que realizan los escolares. Se identificará el número promedio de alumnos que accede a cada propuesta científica durante los días que duró el estudio, atendiendo al género y a la edad.

Con respecto al objetivo $B$, se analizarán las acciones que desarrollan los infantes en las propuestas científicas para evidenciar si éstas promueven experiencias de aprendizaje gratificantes y si favorecen el desarrollo de procesos o habilidades científicas. Las acciones observadas se codifican en función de las categorías de análisis que se muestra en la Tabla 2. Dicha tabla 
presenta dos grupos de categorías que se corresponden con: i) aspectos emocionales de los estudiantes para determinar si las experiencias de aprendizaje son de bienestar y/o favorecedoras; ii) el desarrollo de procesos y habilidades científicas, organizadas según si están relacionadas con la obtención de experiencia, la explicitación de ideas científicas o la evolución de éstas. La definición de estas categorías se establece a partir de trabajos previamente publicados en la literatura (Pedreira y Márquez, 2017a, 2017b) y de las propias observaciones realizadas en el contexto de estudio. Debido al análisis detallado y específico de las acciones observadas que se requiere en este objetivo, se focalizará el estudio únicamente en la propuesta 3 (magnetismo) que, al presentar una afluencia de alumnos elevada, actuará como representantes del conjunto de actividades instaladas en el patio. Para poder analizar las acciones del alumnado, se toman registros durante los días 1, 5 y 12 tras la instalación de dichas propuestas. Dichos registros se organizan en secuencias. Una secuencia se trata de un conjunto de acciones que siguen una única narrativa, con un determinado número de participantes, una intencionalidad, un inicio y un fin (Pedreira y Márquez, 2017a). Para representar los datos recogidos, se realiza un análisis de frecuencias relativas (\%) calculadas a partir del número de veces que se observa una acción determinada, realizada por un estudiante o un conjunto de estudiantes, entre el total de secuencias identificadas.

Con respecto a la fiabilidad del estudio, la recogida y evaluación de los datos fue realizada por dos investigadores que realizaron un estudio piloto previo para entrenarse en la categorización de las secuencias recogidas, alcanzando un acuerdo entre ambos superior al $90 \%$.

\begin{tabular}{|c|c|c|c|}
\hline \multirow{5}{*}{$\begin{array}{r}\text { Aspectos } \\
\text { Emocionales }\end{array}$} & Categorías de estudio & & Definición \\
\hline & \multirow{2}{*}{ Expresiones personales } & Bienestar & $\begin{array}{l}\text { Los estudiantes muestran agrado, emoción, } \\
\text { satisfacción, etc. }\end{array}$ \\
\hline & & No bienestar & $\begin{array}{l}\text { Los infantes dan muestras de descontento, } \\
\text { enfado, etc. }\end{array}$ \\
\hline & \multirow[b]{2}{*}{ Relación entre los estudiantes } & $\begin{array}{c}\text { Acciones } \\
\text { favorecedoras }\end{array}$ & $\begin{array}{l}\text { Los estudiantes se respetan entre ellos, } \\
\text { cooperan, colaboran, hay complicidad, etc. }\end{array}$ \\
\hline & & $\begin{array}{l}\text { Acciones no } \\
\text { favorecedoras }\end{array}$ & $\begin{array}{l}\text { Existe conflicto entre estudiantes, no } \\
\text { comparten el material, se impide la } \\
\text { exploración, etc. }\end{array}$ \\
\hline \multirow{7}{*}{$\begin{array}{l}\text { Procesos de } \\
\text { aprendizaje } \\
\text { científicos }\end{array}$} & \multirow{4}{*}{ Experiencia } & Observación & $\begin{array}{l}\text { Los estudiantes observan el material y la } \\
\text { propuesta sin llegar a interactuar con ella }\end{array}$ \\
\hline & & Manipulación & $\begin{array}{l}\text { Se manipula el material disponible, pero sin } \\
\text { una intencionalidad determinada }\end{array}$ \\
\hline & & $\begin{array}{l}\text { Planteamiento } \\
\text { de retos }\end{array}$ & $\begin{array}{l}\text { Los estudiantes se marcan sus propios retos } \\
\text { u objetivos que intentan conseguir }\end{array}$ \\
\hline & & Rol activo & $\begin{array}{l}\text { Los infantes actúan autónomamente, con } \\
\text { iniciativa propia, sin ayuda de adultos. }\end{array}$ \\
\hline & \multirow{3}{*}{$\begin{array}{l}\text { Explicitación de las ideas: } \\
\quad \text { habilidades cognitivas }\end{array}$} & Clasificación & $\begin{array}{l}\text { Los estudiantes dividen un conjunto de } \\
\text { elementos en clases a partir de un criterio } \\
\text { determinado }\end{array}$ \\
\hline & & Comparación & $\begin{array}{l}\text { Se establecen relaciones de semejanza entre } \\
\text { dos o más elementos }\end{array}$ \\
\hline & & Ordenación & $\begin{array}{l}\text { Los infantes ponen orden a un conjunto de } \\
\text { elementos con un determinado sentido }\end{array}$ \\
\hline
\end{tabular}




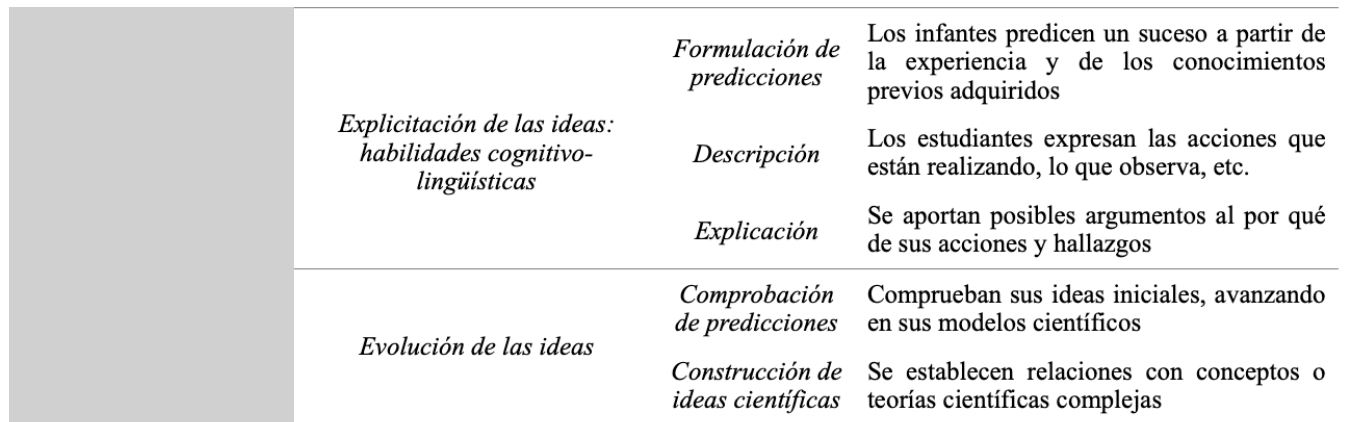

Tabla 2. Instrumento de análisis para estudiar los elementos emocionales y los posibles procesos de aprendizaje derivados de las propuestas científicas instaladas (Pedreira y Márquez, 2017).

\section{Discusión de resultados}

2.1 Estudio del tipo de actividades y comportamiento que el alumnado de educación infantil presenta en el patio escolar antes y después de instalar las propuestas científicas

La Tabla 3 muestra el conjunto de actividades que han sido registradas durante los días previos a la instalación de las propuestas científicas y se han clasificado según su tipología (actividad física, social, cognitiva) y el lugar del patio donde se han observado. Los datos mostrados sirven para realizar una descripción macroscópica de los comportamientos observados en el patio e indican la frecuencia de las actividades registradas en cada zona: 0 para actividades no observadas en una zona concreta, 1 para actividades poco frecuentes y 2 para actividades muy frecuentes.

\begin{tabular}{|c|c|c|c|c|c|c|}
\hline & Tipo de actividad & $\begin{array}{l}\text { Espacio } \\
\text { central } \\
\text { asfaltado }\end{array}$ & $\begin{array}{l}\text { Area } \\
\text { recreativa }\end{array}$ & $\begin{array}{l}\text { Periferia } \\
\text { del patio }\end{array}$ & Arenero & $\begin{array}{l}\text { Espacio } \\
\text { natural }\end{array}$ \\
\hline \multirow{4}{*}{$\begin{array}{l}\text { Actividad } \\
\text { física }\end{array}$} & Juego con balones & 2 & 1 & 1 & 0 & 1 \\
\hline & Uso de bicicletas & 2 & 0 & 1 & 0 & 0 \\
\hline & Escalar, deslizarse & 0 & 2 & 0 & 0 & 0 \\
\hline & Corretear & 2 & 1 & 1 & 0 & 1 \\
\hline \multirow{4}{*}{$\begin{array}{l}\text { Actividad } \\
\text { social }\end{array}$} & $\begin{array}{l}\text { Dialogar entre } \\
\text { compañeros/as }\end{array}$ & 1 & 1 & 2 & 1 & 1 \\
\hline & $\begin{array}{l}\text { Observar otros } \\
\text { compañeros/as }\end{array}$ & 0 & 1 & 2 & 1 & 1 \\
\hline & $\begin{array}{l}\text { Juegos con reglas } \\
\text { (escondite, pilla-pilla, } \\
\text { uso de juegos } \\
\text { tradicionales pintados } \\
\text { en el patio, etc.) }\end{array}$ & 2 & 1 & 3 & 0 & 1 \\
\hline & $\begin{array}{l}\text { Juego de roles (imitar } \\
\text { profesiones, vida de } \\
\text { adultos, etc.) }\end{array}$ & 1 & 1 & 2 & 0 & 0 \\
\hline \multirow[t]{2}{*}{$\begin{array}{l}\text { Actividad } \\
\text { cognitiva }\end{array}$} & $\begin{array}{c}\text { Actividades de } \\
\text { construcción } \\
\text { (construir castillos de } \\
\text { arena, uso de } \\
\text { elementos naturales } \\
\text { con fines creativos, } \\
\text { imaginativos, etc.) }\end{array}$ & 0 & 0 & 0 & 3 & 1 \\
\hline & Explorar el entorno & 0 & 0 & 1 & 1 & 2 \\
\hline
\end{tabular}

Tabla 3. Frecuencia de las actividades observadas en el patio escolar por parte de los participantes. 
Los resultados muestran que la zona central del patio es una de la más ocupada del patio, un área espaciosa donde los estudiantes principalmente realizan actividades de carácter físico como el uso de bicicletas, carreras alrededor de la zona o, principalmente, juego con balones. En esta zona, predomina, principalmente, los estudiantes de mayor edad y de género masculino. Debido a los recursos limitados que se ofrecen (balones, bicicletas), se suelen observar relaciones de poder y hace que estos materiales estén dominados en función del género y también de la edad, obstaculizando la creación de un espacio inclusivo. Estos resultados se replican en otros estudios similares como los publicados por Dyment y O'Connell (2013) o Saldaña (2018). De hecho, el uso del balón tiende a ser la actividad que mayor espacio suele ocupar en los patios y que favorece la segregación de niños o niñas (Saldaña, 2018) y, por ello, algunos centros han empezado a limitar el uso del mismo (Christidou et al., 2013; Saldaña, 2018).

El área recreativa, con una zona de mesas fijas y con diferentes juegos tradicionales pintados en el suelo, es un espacio al que también acceden principalmente los estudiantes de mayor edad pero sin diferencia apreciable de género. Probablemente, la variedad de oportunidades de juego que existen en esta zona facilita que exista dicha diversidad (Paechter y Clark, 2007). Por ejemplo, algunos estudiantes realizan en este espacio actividades de carácter físico mientras que otros realizan juegos de rol. Se observa un bajo uso de los juegos tradicionales pintados en el suelo. Es posible que estos recursos necesiten ir actualizándose con el tiempo para prolongar su interés a lo largo del tiempo y potenciar su imaginación y creatividad (González et al., 2016).

La zona de la periferia del patio es ocupada principalmente por niñas. Este tipo de distribuciones machistas no suelen ser extrañas en los patios escolares y acostumbran a ser las niñas las que más fácilmente tienden a ceder los espacios (Molins-Pueyo, 2012). En los resultados encontrados, se observa que el alumnado que juega en zonas más apartadas realiza actividades más calmadas como dialogar entre ellos, observan o realizan juegos simbólicos.

El arenero es ocupado principalmente por el alumnado de menor edad. Es una zona que permite múltiples acciones y actividades y, de hecho, se observa que los estudiantes utilizan esta zona para construir castillos, explorar las propiedades de la arena, enterrar objetos, etc. Los resultados replican los resultados de Dyment y O'Connell (2013) y se observa como oportunidades este espacio fomenta la creatividad y el juego simbólico y actúa como zona de refugio para los estudiantes que no buscan actividades muy activas.

Finalmente, la zona natural del patio no suele ser muy frecuentada por el alumnado. Se observan alumnos que pasean por la zona explorando árboles o jugando sobre la zona de césped. Probablemente, al ser un espacio con poca diversidad de elementos, influya en la poca afluencia de los estudiantes. En estudios similares, se observa que los espacios naturales suelen ser espacios muy aprovechados por los infantes (Dyment et al., 2009; Dyment y O'Connell, 2013) dando lugar a oportunidades de juego cooperativas, constructivas e imaginativas. Sin embargo, en estos estudios se ha incluido una mayor diversidad de elementos (piedras, troncos, arbustos, árboles), ofreciendo más opciones a los estudiantes y resultando más atractivos para éstos. Este hecho vuelve a ser un ejemplo de la importancia de ofrecer una amplia variedad de elementos para favorecer la inclusión y la diversidad de acciones y actividades de los estudiantes. 
A partir de la incorporación de las diferentes propuestas científicas en el patio se observa un cambio importante en la distribución de los escolares en el patio y en las dinámicas que éstos realizan. La Figura 2 muestra el número promedio de estudiantes que participa en cada actividad a lo largo de los días que duró el estudio. Se observa que gran parte de las actividades que habitualmente suceden en el patio escolar han sido substituidas por intervenciones en algunas de las propuestas científicas. La presencia de las actividades científicas conllevó que más del $70 \%$ del alumnado participara en alguna de las propuestas durante los primeros días del estudio. Dichos datos corroboran el estudio de Paechter y Clark (2007), quienes indican que realizar pequeños cambios en la estructura y organización del patio comporta cambios importantes en el comportamiento y en las actividades que realizan los niños y las niñas. Observando con más detalle cada una de las propuestas, se intuye que los escolares se distribuyeron entre las diferentes actividades en función de su atractivo pero también en función del espacio i material disponible en cada una de las propuestas. En particular, las propuestas magnetismo I y trayectoria del movimiento fueron las que contaron con un mayor número de escolares en todo momento. Probablemente, este hecho sea debido a la novedad y curiosidad que supone para el alumnado interactuar con cierto tipo de materiales (como son los imanes) o a la mayor variedad de retos disponibles. Sin embargo, propuestas que presentan un menor número de variables con las que interactuar, como sonido o magnetismo II, pierden rápidamente el interés por ellas (Figura 2). Este dato confirma la importancia de diseñar propuestas que sean suficientemente abiertas y que ofrezcan posibilidades diversas para que el alumnado pueda plantearse diferentes objetivos y retos, hecho que ayuda a mantener su interés y motivación por la actividad (Pedreira y Márquez, 2016, 2017a). Es importante destacar que el interés del alumnado por las diferentes actividades fue decayendo lentamente a lo largo de los días que duró la investigación (Figura 2). Los escolares, una vez se familiarizan con la propuesta y con las posibilidades que éstas ofrecen, tienden a abandonarla, retomando el tipo de actividades que realizaban previamente a la instalación de las propuestas científicas. Por tanto, parece necesario ir incorporando progresivamente nuevos materiales y retos con en las diferentes propuestas para mantener el interés por ellas. Por ejemplo, en el caso de la propuesta 6 (circuito de agua), se decidió realizar diferentes modificaciones hacia los días finales del estudio y se incorporaron nuevas llaves de paso en el circuito y se modificó el color del agua que circula por su interior para hacerla más visible. Este hecho reforzó el interés y la participación en esta propuesta (González et al., 2016).

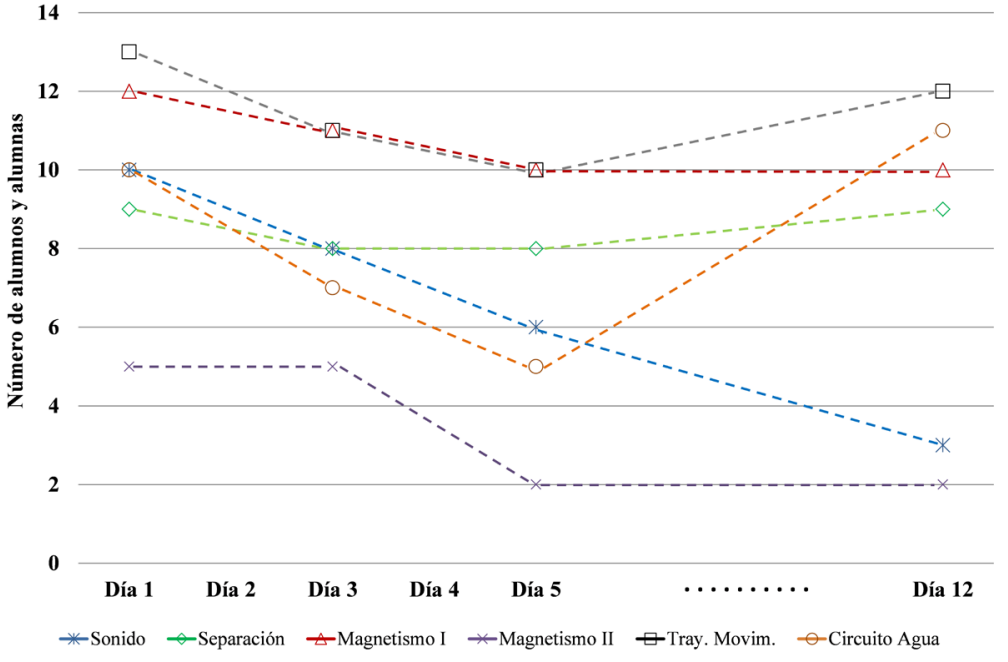

Figura 2. Número promedio de alumnos y alumnas que participa en cada actividad durante los días que duró el estudio. 
La Figura 3 presenta cómo el alumnado se distribuye entre las diferentes propuestas atendiendo a su género y edad. Se evidencia que la intervención realizada en el patio favorece un clima de igualdad entre los dos géneros, observándose una disminución de actividades o sectores del patio monopolizados por un género o edad concreta. En general, no se identifica ninguna propuesta que esté frecuentada mayoritariamente por un género en particular $y$, en todas ellas, interactúa una proporción similar de niños y niñas. En cuanto a la distribución por edad, existen diferencias en algunas de las propuestas. Se intuye que el diferente desarrollo cognitivo y psicomotor del alumnado en función de su edad haya contribuido a estas diferencias observadas (Gopnik et al., 1999). Por ejemplo, las propuestas de sonido o de separación, en las que se desarrollan aspectos principalmente sensoriales, son frecuentadas por los alumnos más pequeños. Sin embargo, en las propuestas de trayectoria de movimiento o de circuito de agua, que implican retos de mayor dificultad y una interacción con un mayor número de elementos mecánicos, se observa una mayor proporción de niños y niñas de 4 y 5 años. Estas evidencias corroboran que los espacios de libre elección favorecen la autonomía y la capacidad de decisión de los alumnos participantes, permitiendo iniciativas diversas del alumnado en función de sus intereses y posibilidades (Pedreira, 2016).
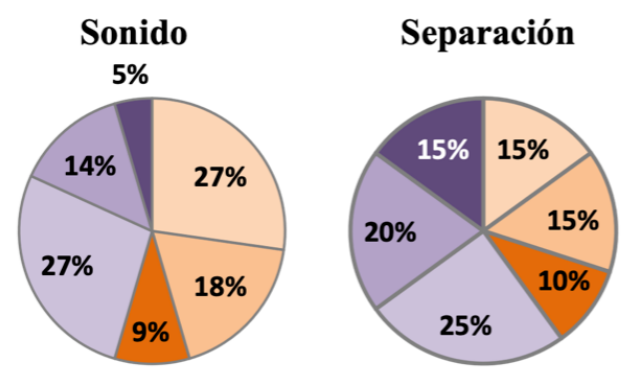

\section{Magnetismo I}

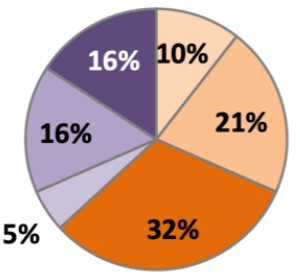

\section{Magnetismo II}

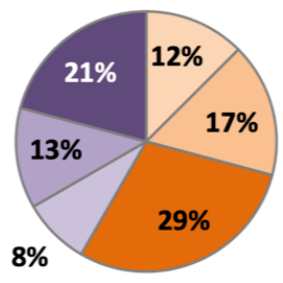

Alumnos 5 años

\section{Trayectoria} movimiento

Alumnas 5 años

Alumnos 4 años
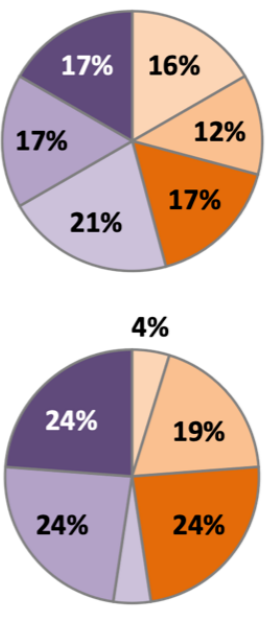

$5 \%$

Circuito de

agua

Alumnas 4 años

Alumnos 3 años

Alumnas 3 años

Figura 3. Distribución promedio, por género y edad, del alumnado que participa en cada propuesta científica. 


\subsection{Analizar el desarrollo de experiencias de aprendizaje gratificantes y el desarrollo de habilidades científicas en el alumnado de educación infantil que participa en las propuestas científicas}

Los resultados derivados del estudio de la propuesta de magnetismo muestr an que la implementación de este tipo de propuestas en el patio de la escuela ha resultado mayormente gratificante para el alumnado (Figura 4). Se presentan expresiones de motivación y satisfacción entre el alumnado participante, sin diferencia entre las edades. Los niños y niñas muestran agrado por las propuestas, hecho que se refleja en sus rostros, gestos de sorpresa o expresiones como "Qué chulo!", "Mira que he descubierto!". Esto ha dado lugar a un clima gratificante en el que el alumnado actúa por iniciativa propia o por imitación (los más pequeños principalmente), estableciéndose también relaciones favorecedoras basadas en la de colaboración, cooperación y complicidad entre los participantes. Por ejemplo, se observa como un grupo de participantes se proponen el reto de conseguir atraer el mayor número posible de objetos metálicos en un mismo imán o de buscar alguna estrategia para conseguir que objetos no magnéticos queden unidos a un imán. Al igual que en otros estudios, el material propuesto favorece notablemente al clima de bienestar (Pedreira y Márquez, 2017b). La introducción de elementos naturales y cuotidianos llama la atención del alumnado y la posibilidad de seleccionar libremente los materiales con los que interactuar contribuye al atractivo de la propuesta. La frecuencia de aparición de expresiones que muestran falta de bienestar es muy baja (Figura 4) y se relacionan con situaciones de frustración o falta de paciencia para conseguir un objetivo ("No lo puedo hacer", "No me sale!"). Aunque no sea objeto de estudio, es importante destacar la presencia del adulto en la propuesta y su participación amable, discreta, atenta y que valora las acciones del alumnado ayuda a promover experiencias de aprendizaje gratificante. De esta manera, el clima emocional afecta el aprendizaje y es importante velar para crear situaciones con climas emocionalmente agradables donde predominen emociones positivas (Lemkow, 2016).

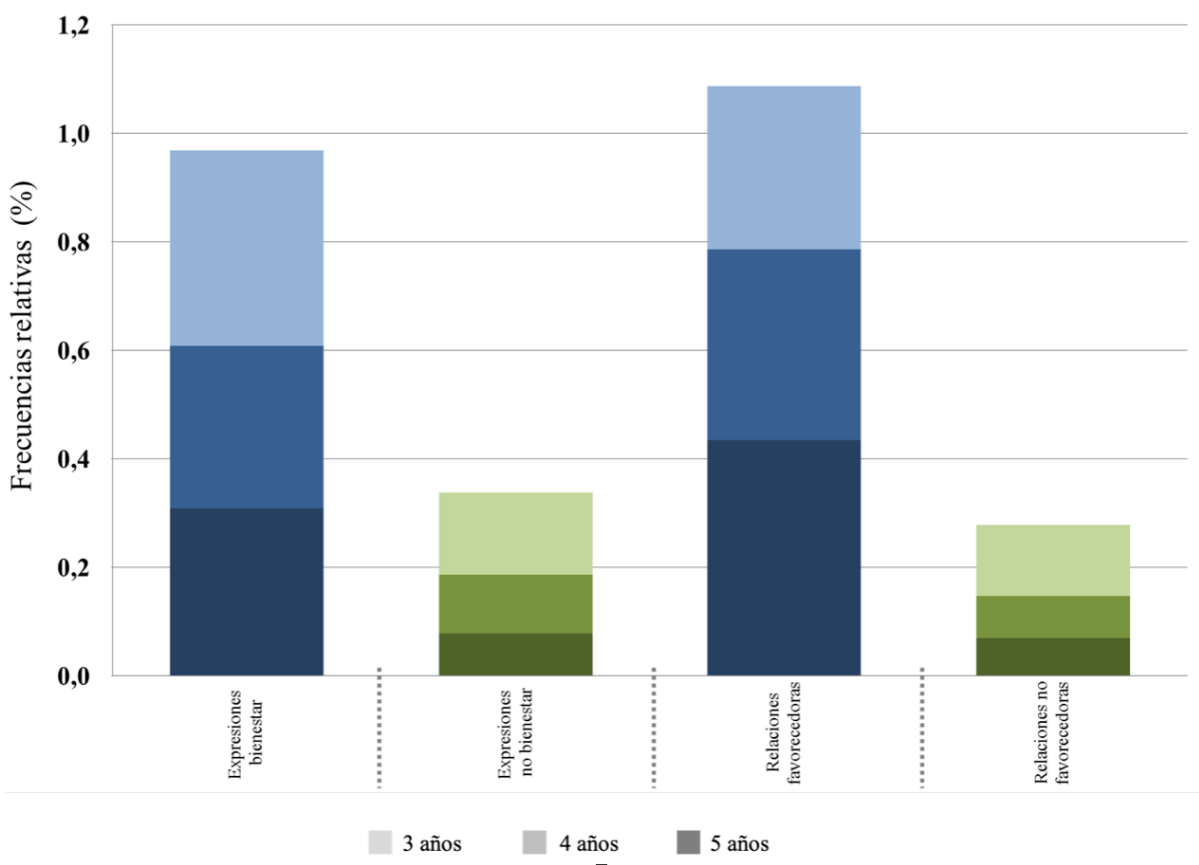

Figura 4. Frecuencia de las experiencias gratificantes y no gratificantes observadas. 
La Figura 5 muestra el desarrollo de las habilidades científicas en la propuesta de magnetismo I. Predominan las acciones exploratorias y el alumnado dedica gran parte del tiempo a manipular e interactuar con los materiales dispuestos para descubrir sus propiedades y reacciones delante de la superficie imantada. Se constata principalmente un comportamiento activo por parte de los participantes y se observa cómo, sin marcarse un objetivo concreto, tienden a tocar el material, identificar sus características, acercarlo a la superficie imantada, comprobar la atracción y la repulsión de determinados objetos, realizar juego simbólico (por ejemplo, entre el material dispuesto había un pincel y algunos participantes lo cogían y hacían ver que pintaban sobre la propuesta), etc. Sin observarse diferencias notables según la edad, este tipo de acciones manipulativas y exploradoras son las más identificadas y destacan por su importancia para empezar a construir ideas sobre el funcionamiento de fenómenos que suceden a nuestro alrededor (Poddiakov, 2011). Con menor frecuencia, y principalmente los niños y niñas de 3 y 4 años, también se muestran observadores de las acciones de sus compañeros. En ocasiones, empiezan utilizando los objetos de cierta manera y, tras observar, acaban dándoles el mismo uso o uno parecido al que los alumnos mayores le dan. Aunque en pocas ocasiones, también se ha constatado el planteamiento de retos por parte del alumnado principalmente mayor. El reto que más se repetía era tratar de conseguir el mayor número de objetos atraídos por un único imán, estableciéndose relaciones de colaboración e incluso competiciones entre diferentes pequeños grupos. La emergencia de este tipo de retos es un elemento a destacar ja que supone la base de procesos indagadores que pueden ampliarse posteriormente en un contexto más formal, dando lugar a nuevas oportunidades con las que construir modelos científicos más avanzados (Quintanilla et al., 2011).

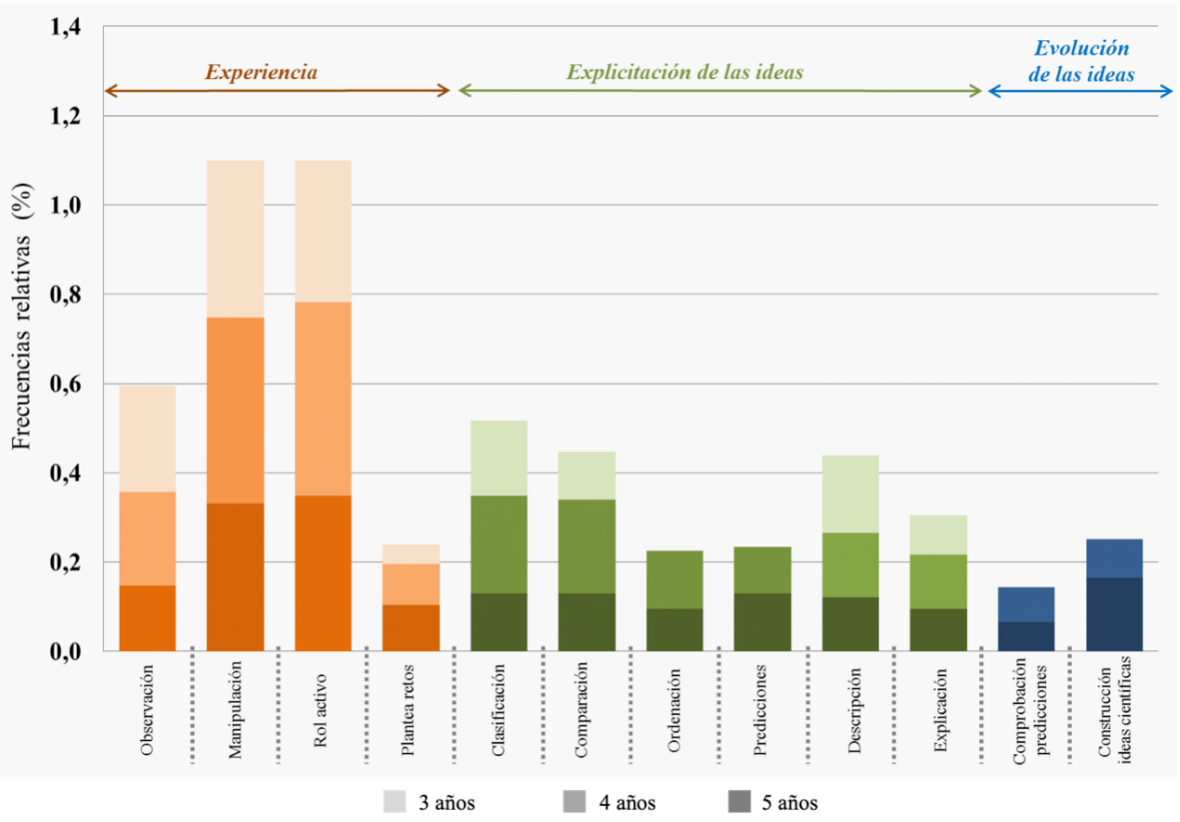

Figura 5. Frecuencia de las diferentes habilidades científicas observadas.

Las habilidades relacionadas con la explicitación de ideas científicas son menos frecuentes y se observan principalmente en el alumnado de 4 y 5 años, en consonancia con su mayor 
desarrollo cognitivo. Las acciones de comparación y clasificación fueron las más identificadas y, por ejemplo, se observa como de manera espontánea algunos alumnos separan los objetos magnéticos de los no magnéticos, buscando semejanzas y diferencias entre ellos. Este tipo de acciones muestran diversos procesos de comunicación que denotan la construcción de conceptos e ideas de carácter científico (Harlen, 2010). Con una frecuencia algo menor, los niños y niñas también han verbalizado ideas científicas relacionadas con las acciones que realizan en la propuesta analizada. El carácter abierto de este tipo de propuestas, las múltiples posibilidades que ofrece y la cotidianidad del material presente favorecen que el alumnado verbalice sus acciones y logros ("los clips no se enganchan", "No, no encaja"), utilizando un lenguaje que puede suponer la base de una futura alfabetización científica más avanzada (Pedreira, 2016). Con menor incidencia, también se identifican argumentos y explicaciones sencillas, en las que el alumnado realiza conexiones con ideas o experiencias anteriores ("la cuchara no se enganchará porque es de madera"). La posibilidad de establecer una experiencia directa con la realidad son clave para promover entre el alumnado dicha incipiente comunicación de ideas científicas, sin la necesidad de realizar todavía razonamientos profundos o precisos.

Finalmente, apenas se han identificado secuencias relacionada con la evolución de ideas científicas. Únicamente entre algunos alumnos de 4 y 5 años aparecen acciones relacionadas con la interpretación de las evidencias recogidas y la construcción de ideas de carácter científico. Nuevamente, el menor desarrollo cognitivo del alumnado de 3 años puede explicar la falta de acciones relacionadas con dichas habilidades científicas (Quintanilla et al., 2011). Por ejemplo, entre las acciones observadas, hay niños y niñas que identifican patrones como la relación que existe los objetos metálicos y la atracción por los imanes ("se pega porque es de metal"). Cuando interactúan con las pinzas de la ropa, la mayoría de los niños las desechan porque creen que no quedarán atraídas por el imán. Pero en las pocas situaciones donde se intuye que el alumno ha encontrado patrones y relaciones, se observa como prueba a unir la parte metálica de la pinza con el imán. La conexión de las evidencias emergidas en el patio con ideas y conceptos científicos concretos es difícil que emerjan espontáneamente. En este tipo de habilidades, se evidencia la necesidad de un soporte por parte del docente que ayude a introducir nuevo conocimiento e impulse las conexiones entre la evidencia y el saber científico, promoviendo la elaboración de modelos más complejos.

\section{Conclusiones}

El presente trabajo analiza la introducción de propuestas didácticas de carácter científico en un patio escolar y los efectos derivados en las acciones que realiza un grupo de niños y niñas de educación infantil. Se trata de propuestas abiertas que ofrecen materiales y recursos con los que descubrir e indagar ideas relacionadas con diferentes ámbitos científicos. La implementación de dicho conjunto de elementos nuevos en el patio ofrece al alumnado nuevas oportunidades para combinar sus actividades de ocio habituales con otras que favorecen la construcción y desarrollo de conceptos y habilidades científicas. Se favorece la diversidad de acciones y comportamientos, la inclusión así como un clima gratificante entre el alumnado participante. Además, las propuestas instaladas ayudan a promover situaciones de aprendizaje en contextos no formales como el patio escolar, fomentándose la exploración de fenómenos, la experimentación o la explicitación de ideas científicas. 


\section{Referencias}

Atmodiwirjo, P. (2013). School ground as environmental learning resources: Teachers' and pupils' perspectives on its potentials, uses and accessibility. International Electronic Journal of Environmental Education, 3(2), 101-119. https://bit.ly/3iy9BoD

Ayotte-Beaudet, J-P., Potvin, P., Lapierre, H. G. y Glackin, M. (2017). Teaching and learning science outdoors in schools' immediate surroundings at K-12 levels: A meta-analysis. EURASIA Journal of Mathematics Science and Technology Education, 13(8), 5343-5363. https://doi.org/gjjqjn

Bisquerra, R. (2004). Metodología de la investigación educativa (1. ${ }^{\mathrm{a}}$ ed.). Editorial La Muralla.

Castro-Pérez, M. y Morales-Ramírez, M. E. (2015). Los ambientes de aula que promueven el aprendizaje, desde la perspectiva de los niños y niñas escolares. Revista Electrónica Educare, 19(3), 1-32. http://dx.doi.org/10.15359/ree.19-3.11

Christidou, V., Tsevreni, I., Epitropou, M. y Kittas, C. (2013). Exploring primary children's views and experiences of the school ground. The case of a Greek school. International Journal of Environmental \& Science Education, 8(1), 59-83. https://bit.ly/3xyRD2e

COSCE (Confederación de Sociedades Científicas de España). (2011). Informe ENCIENDE. Madrid: Confederación de Sociedades Científicas de España.https://www.cosce.org/pdf/Informe_ENCIENDE.pdf

Dyment, J. E., Bell, A. C. y Lucas, A. J. (2009). The relationship between school ground design and intensity of physical activity. Children's Geographies, 7(3), 261-267. http://dx.doi.org/10.1080/14733280903024423

Dyment, J. y O'Connell, T. S. (2013). The impact of playground design on play choices and behaviors of pre-school children. Children's Geographies, 11(3), 263-280. https://doi.org/gj23v5

Epstein, A. S. (2007). Essentials of active learning in preschool: Getting to know the HighScope curriculum. HighScope Press.

Eshach, H. y Fried, M. (2005). Should science be taught in early childhood? Journal of Science Education and Technology, 14(3), 315-336. http://dx.doi.org/10.1007/s10956-005-7198-9

Ferreira, J. M., Karila, K., Muniz, L., Amaral, P. F. y Kupiainen, R. (2018). Children's perspectives on their learning in school spaces: What can we learn from children in Brazil and Finland? International Journal of Early Childhood, 5o(3), 259-277. https://bit.ly/3xtkZis

Fleer, M. y Hardy, T. (2001). Science for children: Developing a personal approach to teaching (2nd ed). Prentice-Hall.

Gallardo, V. B. y Turkienicz, B. (2018). Converting the schoolyard into a learning space: A strategy for public education. Space and Culture, 23(4), 444-261. https://doi.org/ghvh8s

Gómez-Motilla, C. y Ruiz-Gallardo, J. R. (2016). El rincón de la ciencia y la actitud hacia las ciencias en educación infantil. Revista Eureka sobre Enseñanza y Divulgación de las Ciencias, 13(3), 643-666. https://doi.org/gqz6

González, A., Guix, V. y Carreras, A. (2016). La transformación de los patios escolares: una propuesta desde la coeducación. Revista Aula, 255, 67-72. https://bit.ly/3Czg4QU

Gopnik, A., Meltzoff, A. N. y Kuhl, P. K. (1999). The scientist in the crib: Minds, brains, and how children learn. William Morrow \& Co.

Gopnik, A. y Wellman, H. M. (2012). Reconstructing constructivism: Causal models, Bayesian learning mechanisms, and the theory theory. Psychological Bulletin, 138(6), 1085.

http://dx.doi.org/10.1037/a0028044

Harlen, W. (Ed.). (2010). Principles and big ideas of science education. Ashford Colour Press. http://www.interacademies.net/File.aspx?id=25103

Hein, G. (2009) Learning science in informal environments: People, places, and pursuits. Museums \& Social Issues, 4(1), 113-124. http://dx.doi.org/10.1179/msi.2009.4.1.113

Hoyuelos. (2004). La ética en el pensamiento y obra pedagógica de Loris Malaguzzi. Octaedro / Rosa Sensat. 
Kloos, H., Waltzer, T., Maltbie, C., Brown, R.D. y Carr, V. (2018). Inconsistencies in Early Science Education: Can Nature Help Streamline State Standards? Ecopsychologi, 1o(4), 243-258. http://dx.doi.org/10.1089/eco.2018.0042-

Lemkow, G., Brugarolas, I., Cantons, J., Carballo, A. y Mampel, S. (2016). Lab o_6: un espacio de ciencia para la primera infancia. Cuadernos de Pedagogía, (466), 57-59.

http://hdl.handle.net/11162/122263

Lemkow, G. (2016). Lab o-6: espai de ciència, espai neuroeducatiu. Guix d’infantil, (85), 19-22. https://bit.ly/3CzYmfQ

Liguori, L. y Noste, M. I. (2007). Didáctica de las ciencias naturales: enseñar ciencias naturales. Editorial MAD.

Malaguzzi, L. (2005). Els infants, la ciutat i la pluja. En Els cent llenguatges dels infants (p. 215). Rosa Sensat.

Mantzicopoulos, P., Patrick, H. y Samarapungavan, A. (2008). Young children's motivational beliefs about learning science. Early Childhood Research Quarterly, 23(3), 378-394. https://doi.org/bcvf25

Molins-Pueyo, C. (2012). Patios escolares y diversidad sociocultural en Cataluña. Una investigación sobre usos y posibilidades para el juego y el aprendizaje. Papers: Revista de Sociologia, 97(2), 431-460. https://doi.org/10.5565/rev/papers/v97n2.89

National Learning Infraestructure Initiative (2004). Leading the transition from classrooms to learning spaces. https://er.educause.edu/-/media/files/articles/2008/5/nlio447.pdf?la=en

Osborne, J. (2014). Teaching scientific practices: Meeting the challenge of change. Journal of Science Teacher Education, 25(2), 177-196. https://doi.org/gf8rsg

Paechter, C. y Clark, S. (2007). Learning gender in primary playgrounds: Findings from the tomboy identities study. Pedagogy, Culture and Society, 15(3), 317-331. https://doi.org/bqr6cg

Pedreira, M. (2013). Experimentar, quin repte. FUB.

Pedreira, M. y Márquez, C. (2016). Espacios generadores de conocimiento. Cuadernos de Pedagogía, 466, 46-49. https://bit.ly/3xtzt1X

Pedreira, M., \& Márquez, C. (2017a). Espacios de ciencia de libre elección: posibilidades y límites. En M. R. Quintanilla (Ed.), Enseñanza de las ciencia e infancia. Problemáticas y avances de teoría y campo desde Iberoamérica (pp. 151-169). Bellaterra. https://ddd.uab.cat/record/186406

Pedreira, M. y Márquez, C. (2017b). Enabling positive experiences in an informal learning environment for the youngest ages. Journal of Emergent Science, 14, 6-15. https://ddd.uab.cat/record/187282

Poddiakov, N. (2011). Searching, Experimenting and the heuristic structure of a preschool Child's experience. International Journal of Early Years Education, 19(1), 55-63. https://doi.org/frwdth

Pujol, R. M. (2003). Didáctica de las ciencias en la educación primaria. Síntesis.

Quintanilla, M. R., Orellana, M. L. y Daza, S. F. (2011). La ciencia en las primeras edades como promotora de competencias de pensamiento científico. En M. R. Quintanilla y S. F. Daza (Eds.), Enseñanza de las ciencias naturales en las primeras edades: su contribución a la promoción de competencias de pensamiento científico (pp. 59-82). Litodigital.

Rennie, L.J. y Johnston, D.J. (2004). The nature of learning and its implications for research on learning from museums. Science Education, 88, S4-S16. https://doi.org/cgm7n3

Riera, M.A., Ferrer, M. y Ribas, C. (2014). La organización del espacio por ambientes de aprendizaje en la Educación Infantil: significados, antecedentes y reflexiones. RELAdEI. Revista Lationoamericana de Educación Infantil, 3(2), 19-39. https://bit.ly/2U2ZlUv

Rinaldi, C. (2006). Dialogue with Reggio Emilia: Listening, researching and learning. Routledge.

Saçkes, M. (2014). Parents who want their PreK children to have science learning 8S/experiences are outliers. Early Childhood Research Quarterly, (29), 132-143. http://dx.doi.org/10.1016/j.ecresq.2013.11.005

Saldaña, D. (2018). Reorganizar el patio de la escuela, un proceso colectivo para la transformación social. Hábitat y Sociedad, 11, 185-199. https://doi.org/gqz9 
Sevilla, Y. O. (2010). Diseño de espacios educativos significativos para el desarrollo de competencias en la infancia. Revista CS, 71-96. http://dx.doi.org/10.18046/recs.i5.452

Subirats, M. y Tomé, A. (2010). Balones fuera: reconstruir los espacios des de la coeducación. Octaedro. Ting, K. L. y Siew, N. M. (2014). Effects of outdoor school ground lessons on students' science process skills and scientific curiosity. Journal of Education and Learning, 3(4), 96-107. http://dx.doi.org/10.5539/jel.v3n4p96

Tranter, P. J. y Malone, K. (2014). Geographies of environmental learning: An exploration of children's use of school grounds. Children's Geographies, 2(1), 131-155. https://doi.org/bzq2sg 